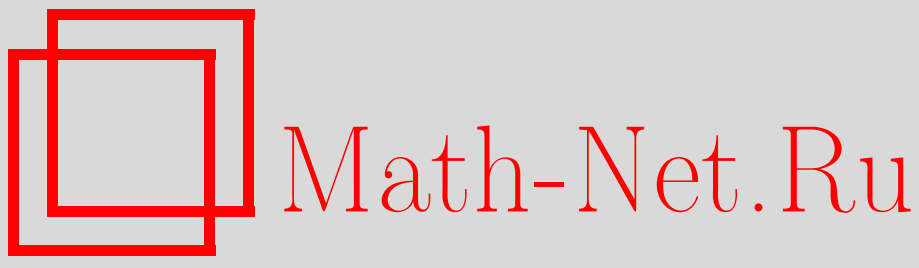

Р. Р. Гонцов, Уточненные неравенства Фукса для систем линейных дифференциальных уравнений, Изв. РАН. Сер. матем., 2004, том 68, выпуск 2, 39-52

DOI: https://doi.org/10.4213/im474

Использование Общероссийского математического портала Math-Net.Ru подразумевает, что вы прочитали и согласны с пользовательским соглашением

http://www . mathnet.ru/rus/agreement

Параметры загрузки:

IP : 3.85 .73 .92

26 апреля 2023 г., $16: 24: 22$ 
УДК 517.531 .57

\author{
P.P. Гонцов
}

\title{
Уточненные неравенства Фукса для систем линейных дифференциальных уравнений
}

\begin{abstract}
Уточняются полученные Э. Корелем неравенства Фукса для системы линейных мероморфных дифференциальных уравнений, заданной на сфере Римана. Неравенства Фукса позволяют оценивать сумму показателей системы по всем ее особым точкам. Уточнение известных неравенств достигается за счет рассмотрения жордановой структуры старшего коэффициента ряда Лорана для матрицы правой части системы в окрестности ее особой точки.

Библиография: 7 наименований.
\end{abstract}

\section{Введение}

Рассмотрим систему вида

$$
\frac{d y}{d z}=B(z) y, \quad B(z)=\frac{B_{-r-1}}{z^{r+1}}+\cdots+\frac{B_{-1}}{z}+B_{0}+\cdots, \quad B_{-r-1} \neq 0,
$$

из $p$ линейных дифференциальных уравнений в окрестности особой точки 0 комплексной плоскости (число $r$ называется рангом Пуанкаре системы в особой точке 0).

Особая точка 0 называется регулярной, если любое решение системы имеет не более чем степенной рост, когда аргумент $z$ стремится к нулю, оставаясь внутри секторов с вершиной в нуле и раствора меньше $2 \pi$. В противном случае особая точка называется иррегулярной.

Если особая точка регулярна, то в пространстве решений сушествует базис (называемый левелевским), элементы которого образуют фундаментальную матрицу $Y(z)$ системы, представимую в виде

$$
Y(z)=U(z) z^{A} z^{E},
$$

где матрица $U(z)$ голоморфна в нуле (голоморфно обратима тогда и только тогда, когда система фуксова в нуле, т. е. когда $r=0), A=\operatorname{diag}\left(\varphi^{1}, \ldots, \varphi^{p}\right)$ - целочисленная матрица нормирований с условием $\varphi^{1} \geqslant \cdots \geqslant \varphi^{p}, E=\frac{1}{2 \pi i} \ln G$, $G$ - верхнетреугольная матрица монодромии системы в нуле и собственные значения $\rho^{j}$ матрицы $E$ удовлетворяют условию

$$
0 \leqslant \operatorname{Re} \rho^{j}<1, \quad j=1, \ldots, p
$$

Работа выполнена при поддержке гранта Минпромнауки (№ НШ-457.2003.01).

$$
\text { (C) Р.Р. Гонцов, } 2004
$$


(подробнее описание нормирований, монодромии и левелевского базиса см. в [3]). Собственные значения $\beta^{j}=\varphi^{j}+\rho^{j}$ матрищы $A+E$ называются левелевскими показателями системы в нуле и характеризуют скорость роста решений в регулярной особой точке.

Случай иррегулярной особой точки сложнее, и подобное разложение справедливо для формальной фундаментальной матрищы $\widehat{Y}(z)$ системы (см. [2]):

$$
\widehat{Y}(z)=\widehat{H}(z) G(z)
$$

где $\widehat{H}(z)$ - обратимый формальньй ряд Лорана в нуле с конечной главной частью и матрица $G(z)$ имеет вид

$$
G(z)=z^{J} U e^{\Theta(z)}
$$

При этом матрищы, входяшие в разложение $G(z)$, представляются одновременно в виде прямой суммы блоков (обозначаемых теми же буквами) и каждый блок имеет вИд

$$
\begin{gathered}
\Theta(z)=\operatorname{diag}\left(q(z) I_{m}, q\left(z e^{2 \pi i}\right) I_{m}, \ldots, q\left(z e^{2 \pi i(s-1)}\right) I_{m}\right), \\
U=\left[\varepsilon^{(j-1)(k-1)} I_{m}\right], \quad 1 \leqslant j, k \leqslant s, \quad \varepsilon=e^{2 \pi i / s}, \\
J=\operatorname{diag}\left(J_{m}, J_{m}+\frac{1}{s} I_{m}, \ldots, J_{m}+\frac{s-1}{s} I_{m}\right),
\end{gathered}
$$

где $q(z)=\widetilde{q}\left(z^{-1 / s}\right), \widetilde{q}(z)$ - многочлен без свободного члена степени не больше $r s$, $s$ - наименьшее натуральное число, для которого $q$ может быть представлен в таком виде, $I_{m}$ - единичная матрица размера $m$ и $J_{m}$ - матрица размера $m$ в жордановой форме, ее собственные значения $\lambda^{j}$ удовлетворяют условию

$$
0 \leqslant \operatorname{Re} \lambda^{j}<\frac{1}{s}, \quad j=1, \ldots, m
$$

Тогда для переменной $t=z^{1 / s}$ (с наименьшим натуральным $s$, для которого $\Theta\left(t^{s}\right)$ не содержит дробных степеней $t$ ) формальная фундаментальная матрица системы может быть представлена в виде

$$
\widehat{Y}(t)=\widehat{F}(t) t^{\Lambda} e^{Q(t)}
$$

где $\widehat{F}(t)$ - обратимьй формальньй ряд Лорана в нуле, $Q(t)=\operatorname{diag}\left(q_{1}(1 / t) I_{1}, \ldots\right.$ $\left.\ldots, q_{l}(1 / t) I_{l}\right), q_{i}$ - многочлен без свободного члена степени не больше $r s, I_{i}-$ единичная матрица размера $m_{i}, \Lambda=\operatorname{diag}\left(\Lambda_{1}, \ldots, \Lambda_{l}\right), \Lambda_{i}$ - блок размера $m_{i}$ в жордановой форме.

Хотя разложение (4) описьвает формальное решение, тем не менее, для любого сектора $S$ (в плоскости переменной $t$ ) с вершиной в нуле и раствора меньше $\pi /(r s)$ найдется настоящее решение $Y_{S}$ такое, что формальный ряд $\widehat{F}(t)$ является асимптотическим в $S$ для функции $Y_{S}(t) e^{-Q(t)} t^{-\Lambda}$ (т. е. тейлоровская часть $\widehat{F}(t)$ является асимптотическим рядом в $S$ для разности $Y_{S}(t) e^{-Q(t)} t^{-\Lambda}$ и главной части $\widehat{F}(t))$. 
Далее, для каждого $i=1, \ldots, l$ рассмотрим пространство $\widehat{X}_{i}$, порожденное столбцами матрищы $\widehat{F}(t) t^{\Lambda_{i}}$. В каждом из пространств $\widehat{X}_{i}$ по аналогии с регулярным случаем определяются понятия формальной монодромии и формальных показателей. Выделим в каждом $\widehat{X}_{i}$ формальный левелевский базис и обозначим через $C_{i}$ матрицу перехода к этому базису. Матрица $C=\operatorname{diag}\left(C_{1}, \ldots, C_{l}\right)$ коммутирует с $Q(t)$. Поэтому, умножив разложение (4) справа на $C$, получим формальную фундаментальную матрищу системы, представимую в окрестности точки 0 плоскости переменной $t$ в виде

$$
\widehat{Y}(t)=\widehat{U}(t) t^{\widehat{A}} t^{\widehat{E}} e^{Q(t)}
$$

где $Q(t)=\operatorname{diag}\left(q_{1}(1 / t) I_{1}, \ldots, q_{l}(1 / t) I_{l}\right)$ - такая же, как в $(4), \widehat{U}(t)-$ формальный ряд Тейлора в нуле, $\widehat{A}=\operatorname{diag}\left(\varphi^{1}, \ldots, \varphi^{p}\right)$ - целочисленная матрица формальных нормирований, $\widehat{E}=\frac{1}{2 \pi i} \ln \widehat{G}, \widehat{G}$ - верхнетреугольная матрища формальной монодромии (блочно-диагонального вида в соответствии с $Q(t)$ ), матрища $t^{\widehat{A}} \widehat{E} t^{-\widehat{A}}$ голоморфна в нуле и собственные значения $\rho^{j}$ матрицы $\widehat{E}$ удовлетворяют условию (3).

Собственные значения $\beta^{j}=\varphi^{j} / s+\rho^{j} / s$ матрищы $\widehat{A} / s+\widehat{E} / s$ называются $ф о р$ мальными показателями системы в нуле (в плоскости переменной $z$ ). Если два формальных решения имеют одинаковую экспоненциальную часть в иррегулярной особой точке, то показатели позволяют различать эти решения по степенному росту.

Если система линейных мероморфных дифференциальных уравнений задана на всей сфере Римана, то возникает вопрос об оценке суммы показателей

$$
\Sigma=\sum_{i=1}^{n} \sum_{j=1}^{p} \beta_{i}^{j}
$$

по всем особым точкам (здесь $\beta_{i}^{1}, \ldots, \beta_{i}^{p}$ - показатели системы в особой точке $a_{i}$ ). Эти оценки называются жеравенствами Фукса и используются при исследовании некоторых вопросов из области аналитической теории дифференциальных уравнений (например, при нахождении кратностей нулей компонент решений системы с особыми точками; см. [4]). До недавнего времени было известно лишь то, что сумма показателей системы с регулярными особыми точками является целым числом, не превосходящим нуль (см. [3]), пока Э. Корелем [5], [6] не были получены следуюшие оценки для порядков функций $\operatorname{det} U(z)$ и $\operatorname{det} \widehat{U}(t)$ в особой точке (из разложений (2) и (5) соответственно):

$$
\begin{gathered}
r \leqslant \operatorname{ord}_{0} \operatorname{det} U(z) \leqslant \frac{p(p-1)}{2} r \\
r s-i_{K}(t) \leqslant \operatorname{ord}_{0} \operatorname{det} \widehat{U}(t) \leqslant \frac{p(p-1)}{2} r s-\frac{1}{2} \operatorname{Irr}_{M}(t),
\end{gathered}
$$

где

$$
i_{K}(t)=\max _{1 \leqslant i \leqslant l} \operatorname{deg} q_{i}, \quad \operatorname{Irr}_{M}(t)=2 \sum_{1 \leqslant i<j \leqslant l} m_{i} m_{j} \operatorname{deg}\left(q_{i}-q_{j}\right)
$$


- соответственно индексы Катца и Мальгранжа иррегулярной особенности системы в точке 0 плоскости переменной $t$. Индексы Катца и Мальгранжа иррегулярной особенности в точке $z=0$ определяются как $i_{K}=i_{K}(t) / s$ и $\operatorname{Irr}_{M}=\operatorname{Irr}_{M}(t) / s$. Из оценок (6) следуют неравенства Фукса:

$$
-\frac{p(p-1)}{2} R+\frac{1}{2} \operatorname{IRR}_{M} \leqslant \Sigma \leqslant-R+I_{K}
$$

где $R$ - сумма рангов Пуанкаре системы по всем особым точкам, а $I_{K}$ и $\mathrm{IRR}_{M}-$ суммы по всем особым точкам индексов Катца и Мальгранжа соответственно.

В настоящей работе оценим порядки функций $\operatorname{det} U(z)$ и $\operatorname{det} \widehat{U}(t)$ в нуле, принимая во внимание структуру жордановой формы матрищы $B_{-r-1}$, и получим уточненные неравенства Фукса (утверждение 5).

\section{§1. Случай регулярной особой точки}

Пусть нуль - регулярная особая точка системы (1) ранга Пуанкаре $r>0$. C учетом ранга матрищы $B_{-r-1}$ оценим порядок в этой точке функции $\operatorname{det} U(z)$ из разложения (2). Для оценки (как в регулярном, так и в иррегулярном случаях) будет использована следующая лемма.

ЛЕмма 1. Для любой голоморфной в окрестности нуля матрицы $U(z)$ существуют верхнетреугольный матричный многочлен $P(z)$ и голоморфно обратимая в окрестности нуля матрича $V(z)$ такие, что

$$
U(z)=V(z) P(z)
$$

Если $U(z)$ - формальный ряд Тейлора в нуле, то утвержсдение леммы остается справедливым после замены голоморфно обратимой матриць $V(z)$ на обратимый формальный ряд Тейлора.

ДокаЗАТЕЛьСтво. Если $\operatorname{det} U(0) \neq 0$, то достаточно положить $V(z)=U(z)$ и $P(z)=I$. Если же $\operatorname{ord}_{0} \operatorname{det} U(z)=l>0$, то $\operatorname{det} U(0)=0$ и существует невырожденная верхнетреугольная матрица $S_{1}$ такая, что столбец с номером $i$ матрицы $U(0) S_{1}$ равен нулю для некоторого $i$. Следовательно,

$$
U(z) S_{1}=U_{1}(z) z^{K_{1}}
$$

где $K_{1}$ - матрица, у которой все элементы равны нулю, кроме элемента с индексом $(i, i)$, который равен единице; $U_{1}(z)$ - голоморфная в окрестности нуля матрица и $\operatorname{ord}_{0} \operatorname{det} U_{1}(z)=l-1$. Поэтому

$$
U(z)=U_{1}(z) z^{K_{1}} S_{1}^{-1}=U_{1}(z) P_{1}(z)
$$

где $P_{1}(z)=z^{K_{1}} S_{1}^{-1}-$ верхнетреугольный многочлен. Повторяя аналогичные рассуждения для матрищы $U_{1}(z)$, получим разложение

$$
U_{1}(z)=U_{2}(z) P_{2}(z)
$$


где $P_{2}(z)$ - верхнетреугольньй многочлен, $U_{2}(z)$ - голоморфиная в окрестности нуля матрица и $\operatorname{ord}_{0} \operatorname{det} U_{2}(z)=l-2$. Окончательно, после $l$ шагов данной процедуры, будем иметь

$$
U(z)=U_{l}(z) P_{l}(z) \ldots P_{1}(z)=V(z) P(z)
$$

где $V(z)=U_{l}(z)$ - голоморфно обратимая в окрестности нуля матрища, $P(z)=$ $P_{l}(z) \ldots P_{1}(z)$ - верхнетреугольный многочлен.

Из леммы 1 сразу же следует, что разложение (2) упрощается:

$$
Y(z)=V(z) P(z) z^{A} z^{E}
$$

(матрицы $V(z)$ и $P(z)$ - такие, как в лемме 1 ), и нам нужно оценить порядок функции $\operatorname{det} P(z)$ в нуле.

ЗАМЕчАНИЕ 1. При голоморфно обратимом в окрестности нуля преобразовании $y=V(z) y^{\prime}$ система (1) переходит в систему с матрицей коэффищиентов

$$
V^{-1}(z) B(z) V(z)-V^{-1}(z) \frac{d V(z)}{d z}
$$

Следовательно, при таком преобразовании ранг Пуанкаре не меняется, а место старшего коэффициента - матрицы $B_{-r-1}$ - занимает матрица $V^{-1}(0) B_{-r-1} V(0)$, т. е. ранг старшего коэффициента также не меняется.

Ввиду этого замечания можно считать, что фундаментальная матрица имеет вид

$$
Y(z)=P(z) z^{A} z^{E}
$$

и тогда матрица $B(z)$ коэффициентов системы $(1)$ верхнетреугольна.

Более того, оказывается, что матрицы $B_{-r-1}, \ldots, B_{-2}$ в ряде Лорана для $B(z)$ нильпотентны. Действительно,

$$
B(z)=\frac{d Y}{d z} Y^{-1}=\frac{d P}{d z} P^{-1}+P \frac{A}{z} P^{-1}+P z^{A} \frac{E}{z} z^{-A} P^{-1},
$$

а так как $P(z)$ - верхнетреугольный многочлен, то диагональные элементы матрицы $\frac{d P}{d z} P^{-1}$ имеют в нуле не более чем простой полюс. Диагональные элементы остальных слагаемых также имеют полюс первого порядка в нуле, а следовательно, это справедливо и для диагональных элементов матрицы $B(z)$.

Рассмотрим преобразование вида $\widetilde{y}=z^{C} y$ с целочисленной матрицей $C=$ $\operatorname{diag}\left(c_{1}, \ldots, c_{p}\right)$ такой, что $c_{1}>\cdots>c_{p} \geqslant 0$. Вследствие (7) это преобразование переводит систему (1) в систему с матрищей коэффициентов

$$
z^{C} B(z) z^{-C}+\frac{C}{z} .
$$

Элементы первого слагаемого этой суммы имеют вид $b_{i j}(z) z^{c_{i}-c_{j}} \quad\left(b_{i j}(z)-\right.$ элементы матрищы $B(z)$ ). Поскольку матрица $B(z)$ верхнетреугольна и ее диагональные элементы имеют простой полюс в нуле, то данное преобразование понижает 
ранг Пуанкаре системы. Если разность соседних диагональных элементов матрицы $C$ равна $r$, то ранг Пуанкаре понижается до нуля. Однако те элементы матрицы $B(z)$, которые имеют полюс порядка $r$ в нуле, достаточно умножить на $z^{r-1}$ (соответствующие им элементы матрищы $B_{-r-1}$ равны нулю).

Пусть $\operatorname{rank} B_{-r-1}=p-k, 1 \leqslant k<p$. Положим

$$
C=\operatorname{diag}((p-1) r,(p-2) r, \ldots, r, 0)+\operatorname{diag}(0, \ldots, k-1),
$$

где $i$-й диагональный элемент во втором слагаемом равен числу

$$
i-1-\operatorname{rank}\left\langle\mathbf{b}_{-r-1}^{1}, \ldots, \mathbf{b}_{-r-1}^{i}\right\rangle, \quad i=1, \ldots, p
$$

$\left(\mathbf{b}_{-r-1}^{j}-j\right.$-й столбец матрицы $\left.B_{-r-1}\right)$. Другими словами, диагональ второго слагаемого в матрице $C$ начинается с нуля, и, далее, элемент с номером $i$ на единицу больше предыдущего только в том случае, если присоединение столбца $\mathbf{b}_{-r-1}^{i}$ не увеличивает ранг системы векторов $\mathbf{b}_{-r-1}^{1}, \ldots, \mathbf{b}_{-r-1}^{i-1}$. Если для некоторых $i<j$ выполнено равенство

$$
c_{i}-c_{j}=(j-i) r-(j-i) \geqslant r-1,
$$

то это означает, что присоединение столбцов $\mathbf{b}_{-r-1}^{i+1}, \ldots, \mathbf{b}_{-r-1}^{j}$ не увеличивает ранг системы векторов $\mathbf{b}_{-r-1}^{1}, \ldots, \mathbf{b}_{-r-1}^{i}$, т. е. элемент с индексом $(i, j)$ матрицы $B_{-r-1}$ равен нулю (вследствие верхнетреугольности и нильпотентности этой матрицы), и функция $b_{i j}(z) z^{c_{i}-c_{j}}$ имеет простой полюс в нуле. В остальных случаях

$$
c_{i}-c_{j} \geqslant(j-i) r-(j-i)+1 \geqslant r
$$

и соответствуюшие функции $b_{i j}(z) z^{c_{i}-c_{j}}$ так же имеют простой полюс в нуле.

Таким образом, преобразование $\widetilde{y}=z^{C} y$ переводит исходную систему (1) в фуксову систему с некоторой фундаментальной матрицей $\widetilde{Y}=\widetilde{U} z^{\widetilde{A}} z^{E}$ такой, что матрица $\widetilde{U}(z)$ голоморфно обратима в нуле (так как получившаяся система фуксова), $\widetilde{A}-$ матрица нормирований этой системы и матрица $z^{\widetilde{A}} E z^{-\widetilde{A}}$ голоморфна в нуле. Тогда

$$
Y(z)=z^{-C} \widetilde{U} z^{\widetilde{A}} z^{E}
$$

Сравнивая это разложение с разложением (8) для матрищы $Y(z)$ и приравнивая порядки определителей в нуле, получим соотношение

$$
\operatorname{tr} A+\operatorname{ord}_{0} \operatorname{det} P(z)=-\operatorname{tr} C+\operatorname{tr} \widetilde{A}
$$

поэтому

$$
\operatorname{ord}_{0} \operatorname{det} P(z)=\operatorname{tr} \widetilde{A}-\operatorname{tr} A-\operatorname{tr} C \text {. }
$$

И теперь, чтобы выяснить, как связаны между собой $\operatorname{tr} A$ и $\operatorname{tr} \widetilde{A}$, воспользуемся следуюшей леммой (доказательство см. в [3]). 
Лемма 2. Пусть фундаментальная матрица $Y(z)$ системь (1) с регулярной особенностью в нуле имеет вид

$$
Y(z)=U(z) z^{L} z^{E}
$$

где матрица $U(z)$ голоморфна в нуле, $L$ - диагональная иелочисленная матрица, E-верхнетреугольная матрица, собственные значения которой удовлетворяют условию (3).

Если матрица $z^{L} E z^{-L}$ голоморфна в нуле, то след матрицы L не превосходит суммы нормирований столбцов матрищы $Y(z)$ в нуле.

Применим эту лемму к системе с фундаментальной матрицей

$$
Y^{\prime}=z^{(p-1) r} Y=z^{(p-1) r I-C} \widetilde{U} z^{\widetilde{A}} z^{E}
$$

и матрищей нормирований $A+(p-1) r I$. Поскольку матрицы $z^{(p-1) r I-C} \widetilde{U}$ и $z^{\widetilde{A}} E z^{-\widetilde{A}}$ голоморфины в нуле, то

$$
\operatorname{tr} \widetilde{A} \leqslant \operatorname{tr} A+p(p-1) r
$$

T. e.

$$
\operatorname{ord}_{0} \operatorname{det} P(z)=\operatorname{tr} \widetilde{A}-\operatorname{tr} A-\operatorname{tr} C \leqslant p(p-1) r-\operatorname{tr} C .
$$

Tак как $\operatorname{tr} C \geqslant p(p-1) r / 2+k(k-1) / 2$, то окончательно получаем следующее утверждение.

УТВЕРЖДЕНИЕ 1. Для порядка функиии $\operatorname{det} U(z)$ в нуле из разложения (2) фундаментальной матрицы системы вида (1) с регулярной особой точкой 0 ранга Пуанкаре $r>0$ справедливо неравенство

$$
\operatorname{ord}_{0} \operatorname{det} U(z) \leqslant \frac{p(p-1)}{2} r-\frac{k(k-1)}{2}
$$

əде $k=p-\operatorname{rank} B_{-r-1}$.

Для получения нижней оценки воспользуемся леммой Соважа из [7], согласно которой для всякой голоморфной в окрестности нуля матрицы $U(z)$ найдется голоморфно обратимая в окрестности нуля матрища $\Gamma(z)$ такая, что

$$
\Gamma(z) U(z)=z^{D} V(z)
$$

где $D=\operatorname{diag}\left(d_{1}, \ldots, d_{p}\right)$ - диагональная целочисленная матрища с условием $d_{1} \geqslant \cdots \geqslant d_{p} \geqslant 0$ и матрица $V(z)$ голоморфно обратима в окрестности нуля. Ввиду замечания 1 преобразование $y^{\prime}=\Gamma(z) y$ не меняет ни ранг Пуанкаре системы, ни ранг старшего коэффициента - матрицы $B_{-r-1}$, поэтому можно считать, что фундаментальная матрица $Y(z)$ системы (1) имеет вид

$$
Y(z)=z^{D} V(z) z^{A} z^{E} .
$$


Тогда для матрицы коэффициентов $B(z)$ справедливо соотношение

$$
\begin{aligned}
B(z) & =\frac{B_{-r-1}}{z^{r+1}}+\cdots=\frac{d Y}{d z} Y^{-1} \\
& =\frac{D}{z}+z^{D}\left(\frac{d V}{d z} V^{-1}+V\left(\frac{A}{z}+z^{A} \frac{E}{z} z^{-A}\right) V^{-1}\right) z^{-D} \\
& =\frac{D}{z}+z^{D} \frac{B_{0}(z)}{z} z^{-D},
\end{aligned}
$$

где $B_{0}(z)$ - голоморфная в окрестности нуля матрища. Из этой формулы следует, что полюс порядка $r+1$ в нуле могут иметь только те элементы матрицы $B(z)$, которые расположены ниже главной диагонали, т. е. $B_{-r-1}-$ нижнетреугольная нильпотентная матрища. Обозначим ее элементы через $b_{i j}$. Если $b_{i j} \neq 0$ для некоторых $i>j$, то $d_{i}-d_{j} \leqslant-r$ и $d_{j} \geqslant r$. Так как существует по меньшей мере $\operatorname{rank} B_{-r-1}$ ненулевых столбцов матрицы $B_{-r-1}$, то существует и $\operatorname{rank} B_{-r-1}$ ненулевых элементов $b_{i j}$ (при $i>j$ ), расположенных в разных столбцах. Следовательно, по меньшей мере для $\operatorname{rank} B_{-r-1}$ чисел $d_{j}$ справедлива оценка $d_{j} \geqslant r$, и тогда $\operatorname{tr} D \geqslant \operatorname{rank} B_{-r-1} r$. Поскольку $\operatorname{ord}_{0} \operatorname{det} U(z)=\operatorname{tr} D($ матрицы $\Gamma(z)$ и $V(z)$ голоморфно обратимы в нуле), то получаем следуюшее утверждение.

УТВЕРЖДЕНИЕ 2. Для порядка функиии $\operatorname{det} U(z)$ в нуле из разложения (2) фундаментальной матрицы системы вида (1) с регулярной особой точкой 0 ранга Пуанкаре $r>0$ справедливо неравенство

$$
\operatorname{ord}_{0} \operatorname{det} U(z) \geqslant \operatorname{rank} B_{-r-1} r .
$$

\section{§2. Случай иррегулярной особой точки}

Пусть теперь нуль - иррегулярная особая точка системы (1) и собственные значения матрищы $B_{-r-1}$ суть $\lambda^{1}, \ldots, \lambda^{h}$.

Сформулируем следующее утверждение о порядке формального ряда Тейлоpa $\operatorname{det} \widehat{U}(t)$ в нуле из разложения (5) формальной фундаментальной матрицы системы.

УТВеРЖДЕНИЕ 3 . Для величинь $\operatorname{ord}_{0} \operatorname{det} \widehat{U}(t)$ справедлива следующая оцен$\kappa a$ :

$$
\operatorname{ord}_{0} \operatorname{det} \widehat{U}(t) \leqslant \frac{p(p-1)}{2} r s-\frac{1}{2} \operatorname{Irr}_{M}(t)-\sum_{j=1}^{h} \frac{k_{j}\left(k_{j}-1\right)}{2} s,
$$

əде $k_{j}=\operatorname{dim} \operatorname{ker}\left(B_{-r-1}-\lambda^{j} I\right), \quad j=1, \ldots, h$.

ДОКАЗАТЕЛЬСТво. Воспользуемся индукцией по $p$ - размеру системы.

В случае $p=1$ неравенство преобразуется в равенство нулю, что верно. Действительно, тогда все коэффициенты $B_{i}$ - числа и фундаментальное решение записывается в виде

$$
y(z)=e^{-B_{-r-1} /\left(r z^{r}\right)-\cdots-B_{-2} / z} z^{B_{-1}} e^{B_{0} z+\cdots}=u(z) z^{B_{-1}} e^{Q(1 / z)},
$$


где $Q$ - многочлен степени $r$, а $u(z)$ - голоморфно обратимая в окрестности нуля функция, т. е. $\operatorname{ord}_{0} u(z)=0$.

Предположив, что утверждение справедливо для систем размера меньше $p$, докажем его для системы размера $p$. Возможны два варианта относительно матрищы $B_{-r-1}$ :

1) матрица $B_{-r-1}$ имеет по меньшей мере два различных собственных значения;

2) собственное значение $\lambda$ - единственное собственное значение матрищы $B_{-r-1}$.

Рассмотрим эти случаи.

Случай 1). В этом случае существует постоянная невырожденная матрища $S$ такая, что матрища $S^{-1} B_{-r-1} S$ является прямой суммой двух блоков размеров $m$ и $p-m$ с различными собственными значениями (в количестве $h_{1}$ и $h-h_{1}$ соответственно), что позволяет воспользоваться следующей леммой (доказательство см. в [1]).

ЛЕмма 3 (о расщеплении). Пусть стариий коэффиииент $B_{-r-1}$ cuстемы (1) имеет блочно-диагональный вид $B_{-r-1}=\operatorname{diag}\left(B_{-r-1}^{11}, B_{-r-1}^{22}\right)$ и блоки $B_{-r-1}^{11}, B_{-r-1}^{22}$ не имеют общих собственных значений.

Тогда существует обратимое формальное аналитическое преобразование $y=\widehat{T}(z) y^{\prime}, \widehat{T}(0)=I$, переводящее систему $(1)$ в систему с (вообще говоря, формальной) матрицей коэффициентов $B^{\prime}(z)=\operatorname{diag}\left(B^{11}(z), B^{22}(z)\right)$ блочно-диагонального вида (в соответствии с $B_{-r-1}$ ).

ЗАмЕчАниЕ 2 . Поскольку $\widehat{T}(0)=I$, то преобразование $\widehat{T}(z)$ не меняет ни старший коэффициент системы, ни порядок формального определителя матрицы $\widehat{U}(t)$ из разложения (5).

Считаем, что матрица $B_{-r-1}$ уже блочно-диагональна (этого можно добиться преобразованием $y=S y^{\prime}$ ). Применив лемму о расщеплении (и учитывая замечание 2), приведем систему (1) к блочно-диагональному виду с двумя блоками размеров $m$ и $p-m$. Матрища $\widehat{U}(t)$ из разложения $(5)$ формальной фундаментальной матрицы получившейся системы имеет соответствующий блочно-диагональный вид

$$
\widehat{U}(t)=\operatorname{diag}\left(\widehat{U}_{1}(t), \widehat{U}_{2}(t)\right)
$$

Пусть формальные фундаментальные матрищы систем, отвечающих этим двум блокам, содержат соответственно $l_{1}$ и $l-l_{1}$ многочленов матрищы $Q(t)$ из разложения (5) (разным собственным значениям соответствуют разные многочлены). Применяя предположение индукции к этим системам размеров $m$ и $p-m$, получим

$$
\begin{aligned}
& \operatorname{ord}_{0} \operatorname{det} \widehat{U}(t)=\operatorname{ord}_{0} \operatorname{det} \widehat{U}_{1}(t)+\operatorname{ord} 0 \operatorname{det} \widehat{U}_{2}(t) \\
& \leqslant \frac{m(m-1)}{2} r s-\frac{1}{2} \operatorname{Irr}_{M}^{1}(t)-\sum_{j=1}^{h_{1}} \frac{k_{j}\left(k_{j}-1\right)}{2} s \\
& \quad+\frac{(p-m)(p-m-1)}{2} r s-\frac{1}{2} \operatorname{Irr}_{M}^{2}(t)-\sum_{j=h_{1}+1}^{h} \frac{k_{j}\left(k_{j}-1\right)}{2} s .
\end{aligned}
$$


Заметим, что

$$
\begin{aligned}
\frac{1}{2} \operatorname{Irr}_{M}(t)-\frac{1}{2} \operatorname{Irr}_{M}^{1}(t)-\frac{1}{2} \operatorname{Irr}_{M}^{2}(t) & =\sum_{i=1}^{l_{1}} \sum_{j=l_{1}+1}^{l} m_{i} m_{j} \operatorname{deg}\left(q_{i}-q_{j}\right) \\
& \leqslant \sum_{i=1}^{l_{1}} \sum_{j=l_{1}+1}^{l} m_{i} m_{j} r s=m(p-m) r s
\end{aligned}
$$

(напомним, что $m_{i}-$ кратность, с которой многочлен $q_{i}$ входит в матрицу $Q(t)$, и степень многочлена $q_{i}$ не превьшает $r s$ ). Поскольку

$$
\frac{m(m-1)}{2} r s+\frac{(p-m)(p-m-1)}{2} r s+m(p-m) r s=\frac{p(p-1)}{2} r s
$$

и

$$
\sum_{j=1}^{h_{1}} \frac{k_{j}\left(k_{j}-1\right)}{2} s+\sum_{j=h_{1}+1}^{h} \frac{k_{j}\left(k_{j}-1\right)}{2} s=\sum_{j=1}^{h} \frac{k_{j}\left(k_{j}-1\right)}{2} s,
$$

то из соотношений (10) следует неравенство (9).

Случай 2). Вследствие леммы 1 (и замечания 1 ) можно считать систему верхнетреугольной и записанной в координате $t$ :

$$
\frac{d y}{d t}=B_{0}(t) y, \quad B_{0}(t)=s t^{s-1} B\left(t^{s}\right)=s\left(\frac{B_{-r-1}}{t^{r s+1}}+\frac{B_{-r} t^{s}}{t^{r s+1}}+\cdots\right) .
$$

В силу (7) скалярное преобразование $y=e^{q(t)} y^{\prime}$ при $q(t)=(-\lambda / r) t^{-r s}$ переводит систему (11) в систему с матрицей коэффициентов

$$
B^{\prime}(t)=B_{0}(t)-\frac{d q}{d t} I=s\left(\frac{B_{-r-1}-\lambda I}{t^{r s+1}}+\frac{B_{-r} t^{s}}{t^{r s+1}}+\cdots\right)
$$

и не меняет индекса Мальгранжа. При этом $B_{-r-1}-\lambda I-$ верхнетреугольная нильпотентная матрища.

Пусть $\operatorname{rank}\left(B_{-r-1}-\lambda I\right)=p-k$. По аналогии с регулярным случаем положим

$$
C=\operatorname{diag}((p-1) s,(p-2) s, \ldots, s, 0)+\operatorname{diag}(0, \ldots, k-1) s,
$$

где $i$-й диагональный элемент во втором слагаемом равен числу

$$
s\left(i-1-\operatorname{rank}\left\langle\widetilde{\mathbf{b}}_{-r-1}^{1}, \ldots, \widetilde{\mathbf{b}}_{-r-1}^{i}\right\rangle\right), \quad i=1, \ldots, p
$$

$\left(\widetilde{\mathbf{b}}_{-r-1}^{j}-j\right.$-й столбец матрицы $\left.B_{-r-1}-\lambda I\right)$.

Тогда замена $\widetilde{y}=t^{C} y^{\prime}$ переводит систему с матрицей коэффициентов $B^{\prime}(t)$ в систему ранга Пуанкаре $r s-s$ в нуле (по тем же соображениям, что и в регулярном случае). Формальная фундаментальная матрица этой системы, соответствуюшая разложению (5), имеет вид

$$
\widetilde{Y}(t)=\widetilde{U}(t) t^{\widetilde{A}} t^{\widehat{E}} e^{Q(t)-q(t) I},
$$


где $\widetilde{U}(t)$ - формальньй ряд Тейлора в нуле, $\widetilde{A}$ - матрица формальньх нормирований получившейся системы и матрища $t^{\widetilde{A}} \widehat{E} t^{-\widetilde{A}}$ голоморфна в нуле. При этом вследствие известных оценок (6) имеем

$$
\operatorname{ord}_{0} \operatorname{det} \widetilde{U}(t) \leqslant \frac{p(p-1)}{2}(r s-s)-\frac{1}{2} \operatorname{Irr}_{M}(t)
$$

Формальная фундаментальная матрица $\widehat{Y}^{\prime}(t)$ помимо разложения

$$
\widehat{Y}^{\prime}(t)=\widehat{U}(t) t^{\widehat{A}} t^{\widehat{E}} e^{Q(t)-q(t) I}
$$

представляется в виде

$$
\widehat{Y}^{\prime}(t)=t^{-C} \widetilde{U}(t) t^{\widetilde{A}} t^{\widehat{E}} e^{Q(t)-q(t) I}
$$

откуда следует соотношение

$$
\operatorname{ord}_{0} \operatorname{det} \widehat{U}(t)+\operatorname{tr} \widehat{A}=-\operatorname{tr} C+\operatorname{ord}_{0} \operatorname{det} \widetilde{U}(t)+\operatorname{tr} \widetilde{A} .
$$

Применим лемму 2 (которая допускает естественное обобщение на иррегулярньй случай) к системе с формальной фундаментальной матрицей

$$
\widehat{Y}^{\prime \prime}(t)=t^{(p-1) s} \widehat{Y}^{\prime}(t)=t^{(p-1) s I-C} \widetilde{U}(t) t^{\widetilde{A}} t^{\widehat{E}} e^{Q(t)-q(t) I}
$$

и матрицей формальных нормирований $\widehat{A}+(p-1) s I$. Матрица $t^{\widetilde{A}} \widehat{E} t^{-\widetilde{A}}$ голоморфна в нуле, a $t^{(p-1) s I-C} \widetilde{U}(t)$ является формальным рядом Тейлора в нуле, поэтому

$$
\operatorname{tr} \widetilde{A} \leqslant \operatorname{tr} \widehat{A}+p(p-1) s .
$$

Поскольку $\operatorname{tr} C \geqslant p(p-1) s / 2+k(k-1) s / 2$, то окончательно из $(13)$, с учетом неравенств (12) и (14), получаем

$$
\begin{aligned}
\operatorname{ord}_{0} \operatorname{det} \widehat{U}(t) & =\operatorname{ord}_{0} \operatorname{det} \widetilde{U}(t)+\operatorname{tr} \widetilde{A}-\operatorname{tr} \widehat{A}-\operatorname{tr} C \\
& \leqslant \frac{p(p-1)}{2}(r s-s)-\frac{1}{2} \operatorname{Irr}_{M}(t)+p(p-1) s-\frac{p(p-1)}{2} s-\frac{k(k-1)}{2} s \\
& =\frac{p(p-1)}{2} r s-\frac{1}{2} \operatorname{Irr}_{M}(t)-\frac{k(k-1)}{2} s .
\end{aligned}
$$

Таким образом, доказательство утверждения 3 завершено.

Нижняя оценка для $\operatorname{ord}_{0} \operatorname{det} \widehat{U}(t)$ в случае иррегулярной особой точки может быть получена аналогично тому, как это было получено в случае регулярной особенности.

УТВЕРЖДЕНИЕ 4. Для порядка формального ряда $\operatorname{det} \widehat{U}(t)$ в нуле из разложения (5) формальной фундаментальной матрицы системь вида (1) с иррегулярной особой точкой 0 ранга Пуанкаре $r>0$ справедливо неравенство

$$
\operatorname{ord}_{0} \operatorname{det} \widehat{U}(t) \geqslant \operatorname{rank} B_{-r-1}\left(r s-i_{K}(t)\right) \text {. }
$$


ДокаЗАТЕЛЬСтво. Можно показать, что функции $q_{j}(1 / t)$, составляющие матрищу $Q(t)$ из разложения $(5)$, имеют вид $q_{j}(1 / t)=\left(-\lambda_{i} /(r s)\right) t^{-r s}+\cdots\left(\lambda_{i}-\right.$ собственные значения матрищы $B_{-r-1}, i=i(j)$ сюръективно). Поэтому неравенство $i_{K}(t)<r s$ выполняется только в том случае, если матрица $B_{-r-1}$ нильпотентна, в противном случае $i_{K}(t)=r s$ и доказьваемая оценка (15) верна.

Итак, будем считать, что матрица $B_{-r-1}$ нильпотентна, а формальная фундаментальная матрица $\widehat{Y}(t)$ системы вследствие формального аналога леммы Соважа и замечания 1 имеет вид

$$
\widehat{Y}(t)=t^{D} \widehat{V}(t) t^{\widehat{A}} t^{\widehat{E}} e^{Q(t)},
$$

где $D=\operatorname{diag}\left(d_{1}, \ldots, d_{p}\right)$ - целочисленная матрица, $d_{1} \geqslant \cdots \geqslant d_{p} \geqslant 0, \widehat{V}(t)-$ обратимый в нуле формальный ряд Тейлора.

Для матрицы коэффициентов системы (в координате $t$ ) выполнено соотношение

$$
\begin{aligned}
s t^{s-1} B\left(t^{s}\right) & =\frac{s B_{-r-1}}{t^{r s+1}}+\cdots=\frac{d \widehat{Y}}{d t} \widehat{Y}^{-1} \\
& =\frac{D}{t}+t^{D}\left(\frac{d \widehat{V}}{d t} \widehat{V}^{-1}+\widehat{V}\left(\frac{\widehat{A}}{t}+t^{\widehat{A}} \frac{\widehat{E}}{t} t^{-\widehat{A}}+\frac{d Q}{d t}\right) \widehat{V}^{-1}\right) t^{-D} \\
& =\frac{D}{t}+t^{D} \frac{B_{0}(t)}{t^{i_{K}(t)+1}} t^{-D},
\end{aligned}
$$

где $B_{0}(t)$ - голоморфная в окрестности нуля матрица.

Поскольку $i_{K}(t)+1<r s+1$, то $B_{-r-1}$ - нильпотентная нижнетреугольная матрица и неравенство (15) вытекает из тех же соображений, что и в регулярном случае (с той лишь разницей, что $B_{0}(t)$ делится не на $t$, а на $\left.t^{i_{K}(t)+1}\right)$.

\section{§ 3. Неравенства Фукса}

Рассмотрим теперь систему

$$
\frac{d y}{d z}=B(z) y
$$

из $p$ линейных мероморфных дифференциальных уравнений, заданную на всей сфере Римана и имеющую особенности в точках $a_{1}, \ldots, a_{n}$ и ранги Пуанкаре $r_{1}, \ldots, r_{n}$ в этих точках соответственно. Разложение в ряд Лорана матрищы $B(z)$ в окрестности точки $a_{i}$ имеет вид

$$
B(z)=\frac{B_{-r_{i}-1}^{i}}{\left(z-a_{i}\right)^{r_{i}+1}}+\cdots+\frac{B_{-1}^{i}}{z-a_{i}}+B_{0}^{i}+\cdots, \quad B_{-r_{i}-1}^{i} \neq 0
$$

Для определенности будем считать, что особенности системы - иррегулярные и каждой точке $a_{i}$ соответствует матрища $\widehat{A}_{i} / s_{i}+\widehat{E}_{i} / s_{i}$, собственные значения которой являются формальными показателями системы в этой точке (матрищы $\widehat{A}_{i}$ и $\widehat{E}_{i}$ взяты из разложения вида $(5)$

$$
\widehat{Y}_{i}(t)=\widehat{U}_{i}(t) t^{\widehat{A}_{i}} t^{\widehat{E}_{i}} e^{Q_{i}(t)}
$$


некоторой формальной фундаментальной матрицы $\widehat{Y}_{i}(t)$ системы в окрестности точки 0 плоскости переменной $\left.t=\left(z-a_{i}\right)^{1 / s_{i}}\right)$; в случае регулярной особенности показателями будут классические показатели Левеля.

Формальная фундаментальная матрица $\widehat{Y}_{i}(t)$ удовлетворяет уравнению

$$
\frac{d \widehat{Y}_{i}}{d t}=s_{i} t^{s_{i}-1} B\left(a_{i}+t^{s_{i}}\right) \widehat{Y}_{i}
$$

поэтому вследствие формулы Лиувилля для однозначной мероморфной формы $d \ln \operatorname{det} \widehat{Y}_{i}(t)$ справедливо соотношение

$$
\begin{aligned}
d \ln \operatorname{det} \widehat{Y}_{i}(t) & =\operatorname{tr} s_{i} t^{s_{i}-1} B\left(a_{i}+t^{s_{i}}\right) d t \\
& =\operatorname{tr}\left(\frac{s_{i} B_{-r_{i}-1}^{i}}{t^{r_{i} s_{i}+1}}+\cdots+\frac{s_{i} B_{-1}^{i}}{t}+s_{i} B_{0}^{i} t^{s_{i}-1}+\cdots\right) d t,
\end{aligned}
$$

откуда следует, что

$$
\operatorname{res}_{0} d \ln \operatorname{det} \widehat{Y}_{i}(t)=s_{i} \operatorname{tr} B_{-1}^{i}=s_{i} \operatorname{res}_{a_{i}} \operatorname{tr} B(z) d z .
$$

Однако

$$
\begin{aligned}
\operatorname{res}_{0} d \ln \operatorname{det} \widehat{Y}_{i}(t) & =\operatorname{res}_{0} d \ln \operatorname{det} \widehat{U}_{i}(t)+\operatorname{res}_{0} d \ln t^{\operatorname{tr}\left(\widehat{A}_{i}+\widehat{E}_{i}\right)} \\
& =\operatorname{ord}_{0} \operatorname{det} \widehat{U}_{i}(t)+\operatorname{tr}\left(\widehat{A}_{i}+\widehat{E}_{i}\right)
\end{aligned}
$$

поэтому

$$
\operatorname{res}_{a_{i}} \operatorname{tr} B(z) d z=\frac{1}{s_{i}} \operatorname{ord}_{0} \operatorname{det} \widehat{U}_{i}(t)+\operatorname{tr}\left(\frac{\widehat{A}_{i}}{s_{i}}+\frac{\widehat{E}_{i}}{s_{i}}\right) .
$$

Из теоремы о сумме вычетов, примененной к форме $\operatorname{tr} B(z) d z$, получаем соотношение для суммы $\Sigma=\sum_{i=1}^{n} \operatorname{tr}\left(\widehat{A}_{i} / s_{i}+\widehat{E}_{i} / s_{i}\right)$ показателей системы по всем особым точкам:

$$
\Sigma=-\sum_{i=1}^{n} \frac{1}{s_{i}} \operatorname{ord}_{0} \operatorname{det} \widehat{U}_{i}(t)
$$

Пусть для каждого $i=1, \ldots, n$ собственные значения матрицы $B_{-r_{i}-1}^{i}$ суть $\lambda_{i}^{1}, \ldots, \lambda_{i}^{h_{i}}$ и

$$
k_{i, j}=\operatorname{dim} \operatorname{ker}\left(B_{-r_{i}-1}^{i}-\lambda_{i}^{j} I\right), \quad j=1, \ldots, h_{i} .
$$

Обозначим через $K_{i}$ сумму $\sum_{j=1}^{h_{i}} k_{i, j}\left(k_{i, j}-1\right) / 2$. Тогда из утверждений 3 и 4 вытекает следуюшая оценка.

УТВеРЖДДЕИЕ 5. Для суммы показателей $\Sigma$ системы (16) справедливы неравенства

$$
-\frac{p(p-1)}{2} \sum_{i=1}^{n} r_{i}+\frac{1}{2} \sum_{i=1}^{n} \operatorname{Irr}_{M, i}+\sum_{i=1}^{n} K_{i} \leqslant \Sigma \leqslant-\sum_{i=1}^{n} \operatorname{rank} B_{-r_{i}-1}^{i}\left(r_{i}-i_{K, i}\right),
$$


әде $\operatorname{Irr}_{M, i}$ u $i_{K, i}$ - соответственно индексы Мальгранжа и Катиа в особой mочке $a_{i}$.

Таким образом, уточнение неравенств Фукса, полученных Э. Корелем, происходит за счет добавления к нижней оценке слагаемого $\sum_{i=1}^{n} K_{i}$ и умножения на $\operatorname{rank} B_{-r_{i}-1}^{i}$ каждого слагаемого $-r_{i}+i_{K, i}$ из верхней оценки.

С учетом утверждений 1 и 2 неравенства (17) справедливы и для системы с регулярными особыми точками. При этом $\operatorname{Irr}_{M, i}=i_{K, i}=0$ для каждой особой точки (фундаментальная матрица не имеет экспоненциальную часть) и матрищы $B_{-r_{i}-1}^{i}$ нильпотентны, т. е. $K_{i}=k_{i}\left(k_{i}-1\right) / 2$, где $k_{i}=p-\operatorname{rank} B_{-r_{i}-1}^{i}$. Следовательно,

$$
-\frac{p(p-1)}{2} \sum_{i=1}^{n} r_{i}+\sum_{i=1}^{n} K_{i} \leqslant \Sigma \leqslant-\sum_{i=1}^{n} \operatorname{rank} B_{-r_{i}-1}^{i} r_{i}
$$

для системы (16) с регулярными особенностями.

В завершение автор выражает особую благодарность А. А. Болибруху за ключевые советы по уточнению неравенств Фукса и помощь в написании работы, а также за предоставление аналитических методов, разработанных во время участия Андрея Андреевича в школе по асимптотическим рядам, проходившей несколько лет назад в Монреале.

\section{Список литературы}

1. Balser $W$. Formal power series and linear systems of meromorphic ordinary differential equations. N.Y.: Springer-Verlag, 2000.

2. Balser W., Jurkat W.B., Lutz D.A. A general theory of invariants for meromorphic differential equations. I. Formal invariants // Funk. Evac. 1979. V. 22. P. 197-221.

3. Болибрух A. A. 21-я проблема Гильберта для линейных фуксовых систем // Тр. Матем. ин-та им. В. А. Стеклова РАН. 1994. Т. 206.

4. Болибрух A. A. Кратности нулей компонент решений системы с регулярными особыми точками // Тр. Матем. ин-та им. В. А. Стеклова РАН. 2002. Т. 236. С. 61-65.

5. Corel E. Inégalités de Fuchs pour les systèmes differéntiels réguliers // C. R. Acad. Sci. Paris. 1999. V. 328. Sér. I. P. 983-986.

6. Corel E. Relations de Fuchs pour les systèmes differéntiels irréguliers // C. R. Acad. Sci. Paris. 2001. V. 333. Sér. I. P. 297-300.

7. Хартман $\Phi$. Обыкновенные дифференциальные уравнения. М.: Мир, 1970.

Поступило в редакцию 11.09.2003 